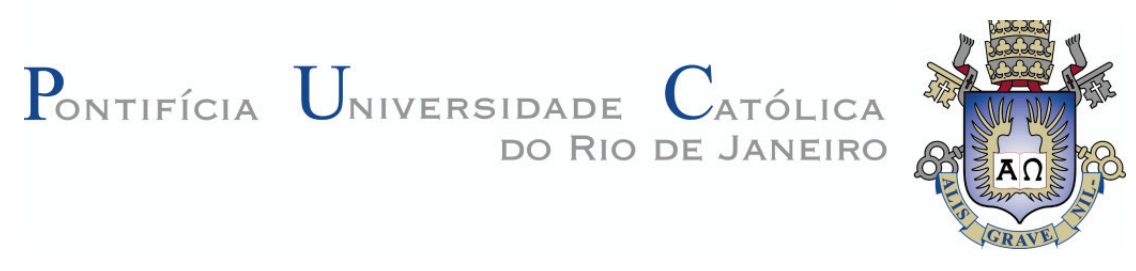

Luis Fernando Paullo Muñoz

\title{
Análise Dinâmica Não Linear de Pórticos com Base Elasto-Plástica sob Ação Sísmica
}

\section{Tese de Doutorado}

Tese apresentada como requisito parcial para obtenção do título de Doutor pelo Programa de Pós-Graduação em Engenharia Civil da PUC-Rio.

Orientador: Prof. Paulo Batista Gonçalves Co-orientador: Prof. Ricardo Azoubel da Mota Silveira 


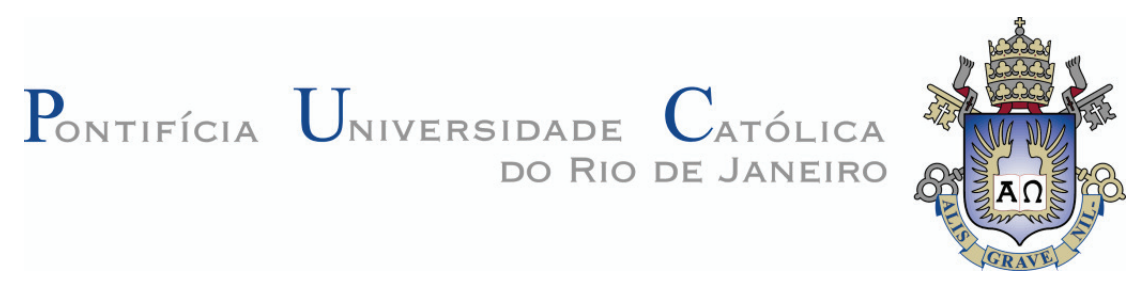

Luis Fernando Paullo Muñoz

\section{Análise Dinâmica Não Linear de Pórticos com Base Elasto-Plástica sob Ação Sísmica}

Tese apresentada como requisito parcial para obtenção do título de Doutor pelo Programa de Pós-Graduação em Engenharia Civil do Departamento de Engenharia Civil do Decanato do CTC da PUC-Rio. Aprovada pela Comissão Examinadora abaixo assinada.

Prof. Paulo Batista Gonçalves Orientador Departamento de Engenharia Civil - PUC-Rio

Prof. Ricardo Azoubel da Mota Silveira Co-orientador Departamento de Engenharia Civil - UFOP

Prof. Sebastião Arthur Lopes de Andrade Departamento de Engenharia Civil - PUC-Rio

Prof. Deane de Mesquita Roehl Departamento de Engenharia Civil - PUC-Rio

Prof. José Guilherme Santos da Silva Universidade do Estado do Rio de Janeiro

Profa . Michéle Schubert Pfeil Universidade Federal do Rio de Janeiro

Prof. José Eugenio Leal Coordenador Setorial do Centro Técnico Científico - PUC-Rio

Rio de Janeiro, 27 de Março de 2015 
Todos os direitos reservados. É proibida a reprodução total ou parcial do trabalho sem autorização da universidade, do autor e do orientador.

\section{Luis Fernando Paullo Muñoz}

Graduou-se em Engenharia Civil no Departamento de Engenharia Civil da UNSAAC (Universidad Nacional de San Antônio Abad Del Cusco), em 2006. Obteve o grau de Mestre em Engenharia Civil pelo Departamento de Engenharia Civil da PUC-Rio em 2010. Atualmente tem continuado com a linha de pesquisa na área de instabilidade e análise dinâmica não linear de estruturas, com o estudo de análise sísmica de pórticos com iteração solo-estrutura.

Ficha Catalográfica

Paullo Muñoz, Luis Fernando

Análise dinâmica não linear de pórticos com base elastoplástica sob ação sísmica / Luis Fernando Paullo Muñoz; orientador: Paulo Batista Gonçalves; co-orientador: Ricardo Azoubel da Mota Silveira. - 2015.

214 f. : il. (color.) ; $30 \mathrm{~cm}$

Tese (doutorado)-Pontifícia Universidade Católica do Rio de Janeiro, Departamento de Engenharia Civil, 2015. Inclui bibliografia

1. Engenharia civil - Teses. 2. Análise dinâmica não linear. 3. Excitação sísmica. 4. Análise em frequência. 5. Instabilidade dinâmica. 6. Métodos de integração no tempo. 7. Método do balanço harmônico. I. Gonçalves, Paulo Batista. II. Silveira, Ricardo Azoubel da Mota. III. Pontifícia Universidade Católica do Rio de Janeiro. Departamento de Engenharia Civil. IV. Título 


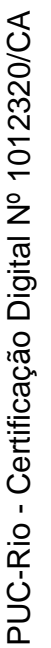

Aos meus pais, Fernando e Rosa. A minha irmã, Yesenia. 


\section{Agradecimentos}

A Deus, por permitir-me culminar mais uma etapa na minha vida.

A minha mãe Rosa e ao meu pai Fernando, a quem devo tudo o conseguido na vida.

A minha irmã Yesenia, que foi e é meu apoio nos momentos mais difíceis.

Ao Professor Paulo B. Gonçalves, pela orientação, serenidade e conhecimentos transmitidos.

Aos Professores Ricardo Silveira e Andréa da Silva pela ajuda e orientação prestada.

Aos professores do setor estruturas do DEC da PUC-Rio, pelo ensino acadêmico e motivação dada ao longo do mestrado.

À UNSAAC (Universidad de San Antônio Abad del Cusco), pela base académica necessária para minha formação.

Aos colegas da PUC-Rio, pela ajuda acadêmica, pessoal e pelos momentos de convívio, em especial aos colegas que começaram esta aventura académica ainda em 2008.

À CNPq e à PUC-Rio, pelo auxílio financeiro.

Ao instituto Tecgraf da PUC-Rio, pela oportunidade de desenvolver outras áreas de conhecimento e sua contribuição na minha estabilidade financeira.

A todas as pessoas que de alguma forma contribuíram com a elaboração deste trabalho. 


\section{Resumo}

Paullo Muñoz, Luis Fernando; Gonçalves, Paulo Batista; Silveira, Ricardo Azoubel da Motta. Análise Dinâmica Não Linear de Pórticos com Base Elasto-plástica sob Ação Sísmica. Rio de Janeiro, 2015. 214p. Tese de Doutorado - Departamento de Engenharia Civil, Pontifícia Universidade Católica do Rio de Janeiro.

A resposta dinâmica de sistemas estruturais não lineares tem sido um item de grande interesse nas pesquisas em engenharia civil. Problemas onde há interação base flexível-estrutura são de grande importância na análise estrutural, já que a maioria das estruturas civis é apoiada sobre sistemas flexíveis (solo ou sistemas de apoio com dissipação de energia). Nesta área, o estudo de sistemas submetidos a ações sísmicas é um tópico relevante, já que estas solicitações têm um grande conteúdo de frequências, o que pode influenciar consideravelmente as respostas da estrutura. Neste contexto, o conhecimento da resposta em frequência de estruturas não lineares sob uma excitação de base é uma ferramenta útil para avaliar os potenciais efeitos de ações sísmicas sobre estes sistemas. Na presente tese é desenvolvida uma metodologia de análise não linear dinâmica de sistemas estruturais reticulados sob excitações de base, considerando não linearidade geométrica e apoios flexíveis, representados por molas unidimensionais, com comportamento elasto-plástico. Através de uma análise paramétrica é avaliada a variabilidade das respostas de sistemas esbeltos submetidos a ações sísmicas reais, sismos artificiais, assim como ações sísmicas sucessivas. O problema no espaço é resolvido pelo método dos elementos finitos. Para a análise em frequência, é apresentada uma metodologia baseada no método do balanço harmônico e no método de Galerkin, juntamente com técnicas de continuação para a obtenção das curvas de ressonância não lineares. O problema no tempo é abordado através da integração das equações de movimento pelos métodos de Runge-Kutta e Newmark, associado ao método de Newton-Raphson.

\section{Palavras-chave}

Análise dinâmica não linear; Excitação sísmica; Análise em frequência; Instabilidade dinâmica; Métodos de integração; Método do balanço Harmônico. 


\section{Abstract}

Paullo Muñoz, Luis Fernando; Gonçalves, Paulo Batista (Advisor); Silveira, Ricardo Azoubel da Motta (Co-advisor). Nonlinear Dynamic Analysis of Frames with Elasto-plastic Base under Seismic Excitation. Rio de Janeiro, 2015. 214p. Doctoral Thesis - Departamento de Engenharia Civil, Pontifícia Universidade Católica do Rio de Janeiro.

The dynamic response of nonlinear structures has been a topic of interest in civil engineering research. Problems in which base-structure interaction is present have a great importance in structural analysis, since most structures rests on flexibel systems (soil or supports with dissipation). In this research area, the study of structures under the action of seismic loads represent a relevant topic, since this kind of excitations may excite several vibration modes and thus influence strongly the dynamic response. In this context, the prediction of the nonlinear structural behavior in frequency domain of structures under base excitation is a useful resource to assess the potential effects of sismic loads on these systems. In this thesis, a methodology for nonlinear dynamic analysis of plane frame structures under base excitation is presented considering geometric nonlinearity and elastic supports represented by elasto-plastic unidimensional springs. Trough a parametric analysis, the variability of the dynamic responses of slender structural systems under the actions of real earthquakes, synthetics earthquakes, as well as the action of multiple earthquakes is assessed. The structural systems here analyzed are discretized in space using a nonlinear finite element formulation. For the response in frequency domain, a scheme based on the Balance Harmonic Method and the Galerkin method, in conjunction with continuation methods, is formulated to obtain the nonlinear resonance curves. The nonlinear dynamic response in the time domain is calculated by direct integration of the equations of motion. For this, the Runge-Kutta method and the Newmark method in association with the iterative Newton-Raphson scheme are employed.

\section{Keywords}

Nonlinear dynamic analysis; Earthquakes; Analysis in frequency domain; Dynamic instability; Direct integration methods; Harmonic Balance method. 


\section{Sumário}

1 Introdução 27

1.1. Considerações Iniciais 27

1.2. Objetivos 28

1.3. Revisão Bibliográfica 28

1.3.1. Não Linearidade Geométrica e Física 28

1.3.2. Aspectos de Dinâmica Estrutural e Análise Sísmica. 31

1.3.3. Consideração de base flexível 34

1.4. Organização e Descrição da Tese 40

2 Formulação Não Linear Estática e Dinâmica no Domínio do Tempo 42

2.1. Formulação do Elemento Finito Não Linear 42

2.1.1. Medidas de deformação não linear 42

2.1.1.1. Formulação Não Linear FN1 43

2.1.1.2. Formulação Não Linear FN2 44

2.1.2. Forças Internas Resultantes e Energia Potencial Total 45

2.1.3. Discretização do sistema por elementos finitos 49

2.1.4. Matriz de rigidez, vetor de forças internas e equilíbrio estático 50

2.1.5. Formulação do elemento com ligação semirrígida 51

2.1.6. Matriz de rotação e montagem do sistema global estático 56

2.2. Formulação para Análise Dinâmica no Domínio do Tempo 57

2.2.1. Energia potencial e equação de movimento 57

2.2.2. Matriz de massa considerando ligações semirrígidas 59

2.2.3. Matriz de amortecimento e sistema global de equilíbrio 60

2.2.4. Equação de movimento para excitação de base 61

2.3. Solução no domínio do Tempo 62 
2.3.1. Frequências naturais e modos de vibração 62

2.3.2. Analise transiente e integração direta no domínio do tempo 64

2.3.3. Métodos de integração para análise linear 65

2.3.3.1. Método implícito de Newmark 65

2.3.3.2. Métodos explícitos de Runge-Kutta 66

2.3.3.3. Integração do sistema dinâmico linear pelos métodos Runge-

Kutta explícitos $\quad 72$

2.3.3.4. Método implícito de Runge-Kutta-Gauss 73

2.3.4. Integração direta para análise não linear 74

2.3.4.1. Método Implícito de Newmark para análise não linear 74

2.3.4.2. Método de Runge-Kutta para análise não linear 75

2.4. Aspectos de Solução Estática Não Linear 77

2.4.1. Método iterativo de Newton-Raphson 78

2.4.2. Método do controle de arco 79

2.5. Consideração de Base Flexível 81

2.5.1. Formulação dinâmica do modelo contínuo com subestruturação 81

2.5.2. Formulação dinâmica com modelos discretos 82

2.5.3. Elemento de Base Elasto-plástica 84

3 Excitação Sísmica 86

3.1. Conceitos Gerais Sobre Sismos e Sismicidade 86

3.1.1. Definição e principais características dos sismos 86

3.1.2. Propagação e tipo de ondas sísmicas 89

3.2. Efeito dos Sismos sobre os Sistemas Estruturais 90

3.3. Formulação da Excitação Sísmica na Análise Estrutural 92

3.3.1. Registro da Aceleração sísmica 92

3.3.2. Potência da excitação sísmica 93

3.4. Geração de Sismos Artificiais 96

3.4.1. Formulação de sismos artificiais 96

3.4.2. Função intensidade e correção da linha base 98

4 Exemplos Numéricos da Análise no Domínio do Tempo 101

4.1. Descrição dos modelos estudados 101

4.1.1. Torre esbelta de três graus de liberdade T-1 101 
4.1.2. Torre esbelta de com massa concentrada no topo T-2 102

4.1.3. Pórtico de dois andares Po-2 103

4.1.4. Pórtico de seis andares Po-6 103

4.1.5. Arco circular abatido Ac-1 104

4.2. Exemplos de sistemas lineares sob excitação harmônica 105

4.2.1. Validação dos métodos de integração 105

4.2.2. Estudo de desempenho e estabilidade 107

4.2.3. Influência de base elástica 110

4.2.3.1. Influência na resposta no tempo 110

4.2.3.2. Influência na ressonância 111

4.3. Exemplos da Análise Não Linear - Excitação Harmônica 113

4.3.1. Influência da não linearidade geométrica 113

4.3.1.1. Validação da integração direta no tempo 113

4.3.1.2. Não linearidade geométrica na ressonância 116

4.3.1.3. Não linearidade geométrica e variação da amplitude da aceleração 118

4.3.1.4. Não linearidade geométrica e variação da rigidez 119

4.3.2. Consideração de base elasto-plástica 121

4.4. Análise Sísmica 123

4.4.1. Geração de sismo artificial 123

4.4.2. Comparação entre as resposta com sismos real e artificial 126

4.4.3. Excitação sísmica e não linearidade geométrica 127

4.4.4. Excitação sísmica e elasto-plasticidade da base 127

5 Formulação Dinâmica Não Linear no Domínio da Frequência 129

5.1. Obtenção de Curvas de Ressonância Através da Análise no Tempo 130

5.2. Método do Balanço Harmônico 131

5.3. Formulação Linear do Equilíbrio Dinâmico na Frequência 133

5.4. Formulação Não Linear do Equilíbrio Dinâmico na Frequência:

Método

do Balanço Harmônico-Galerkin 134 
5.5. Solução do Sistema de Equações Não Lineares na Frequência:

Controle

de Arco

5.6. Não Linearidade Geométrica na Frequência

5.7. Rotação da Matriz de Rigidez Não Linear no Domínio ।

e Montagem do Sistema Global

6 Exemplos Numéricos no Domínio da Frequência

6.1. Resposta em Frequência: Formulação linear

6.1.1. Pilar engastado e livre 145

6.1.2. Torre com massa concentrada 148

6.2. Resposta em Frequência: Formulação Não Linear

6.2.1. Sistema de um grau de liberdade com rigidez não linear quadrática

6.2.2. Influência da intensidade da excitação e não linearidade cúbica 151

6.2.3. Arco bi-engastado: Efeito da não linearidade quadrática

6.3. Efeito de não linearidade geométrica em estruturas esbeltas

6.3.1. Estrutura de galpão com cobertura de duas águas

6.3.1.1. Resposta para solicitação de base 156

6.3.1.2. Resposta para solicitação na direção vertical 158

6.3.1.3. Resposta para solicitação horizontal e vertical simultânea 159

6.3.2. Estrutura de galpão com cobertura em arco 161

6.3.2.1. Resposta para solicitação sísmica horizontal 163

6.3.2.2. Resposta para solicitação sísmica Vertical 164

6.3.2.3. Resposta para solicitação sísmica horizontal e vertical simultâneos

7 Estruturas Submetidas a Sismos Reais e Artificias com Interação Solo-Estrutura

7.1. Solicitações Sísmicas

7.1.1. Solicitações sísmicas reais

7.1.2. Solicitações sísmicas artificiais 
7.2.2. Resposta a sismos artificiais com semelhança de potência

7.2.3. Resposta a sismo artificial com potência concentrada nas regiões de ressonância

7.2.3.1. Obtenção do espectro de potência

7.2.3.2. Resposta a sismos artificias com potência concentrada na ressonância

7.3. Galpão com duas águas:

7.3.1. Resposta a sismos reais

7.3.2. Resposta a sismos artificiais com semelhança de potência

7.3.3. Resposta a sismos artificiais com potência concentrada nas regiões de ressonância.

7.4. Galpão Cobertura em Arco

7.4.1. Respostas máximas em sismos reais e artificias gerados a partir de potências reais

7.4.2. Resposta a sismos artificiais com potência concentrada nas regiões de ressonância.

7.4.3. Resposta a ações sísmicas múltiplas

8 Conclusões e Sugestões

8.1. Conclusões

201

8.2. Sugestões para Trabalhos Futuros

203

9 Referências Bibliográficas 


\section{Listas de Figuras}

Figura 1.1 - Referenciais Lagrangianos. Ref. Silva (2009).

29

Figura 1.2 - Análise não linear geométrica e inelástica de pórtico metálico. Ref. Silva (2009).

Figura 1.3 - Modelos de solo como meios elásticos contínuos.

Ref. Paullo (2010).

Figura 1.4 - Métodos diretos e de sub estruturação na análise de interação solo-estrutura. Ref. Villaverde (2009).

Figura 1.5 - Esquema e modelo de elementos finitos no estudo de interação estrutura-solo-estrutura através de modelo contínuo. Ref. Clouteau et al. (2009).

Figura 1.6 - Sistema discreto de interação base-estrutura. Ref. Wolf (1994).

Figura 1.7 - Representação de solo por sistema de barras unidimensionais.

Ref. Halabian (1994).

Figura 1.8 - Modelos unidimensionais de solo. Esquerda: Sistema de molas

Ref. Miguel e Bouaanani (2011). Direita: Sistema de mola-amortecedor Ref. Ganjavi e Hao (2012).

Figura 2.1 - Comportamento da seção transversal do elemento barra.

Ref. Silva (2009).

Figura 2.3 - Graus de liberdade do elemento finito de viga-coluna. $\quad 49$

Figura 2.4 - Idealização de ligação semirrígida. 51

Figura 2.5 - Sistema auxiliar de barra com molas. 54

Figura 2.6 - Sistema Estrutural submetido a deslocamento de base. 62

Figura 2.7 - Esquema de integração do método de Euler. 67

Figura 2.8 - Aproximação iterativa secante. 76

Figura 2.9 - Aproximação tangente no método de Newton-Raphson. 78 
Figura 2.10 - Aproximação por arco esférico. 80

Figura 2.11 - Sistema base-estrutura (Halabian,2002). 81

Figura 2.12 - Sistema unidimensional discreto solo-estruturé 1985).

Figura 2.13 - Relação de força vs. Deslocamento de mola transli rotacional unidimensional com comportamento elasto-plástico bi(Wolf, 1985).

Figura 3.1 - Localização geográfica do hipocentro e epicentro d€ sismo (https://bussoladeplasma.wordpress.com/tag/epicentro).

Figura 3.2 - Distribuição de atividades sísmicas no mundo (http://www.visionlearning.com).

Figura 3.3 - Tipo e forma de propagação das ondas sísmicas no solo. 90

Figura 3.4 - Falha do solo por ação sísmica: a) fratura de solo em estrutura de estrada devida à vibração excessiva no terremoto de Kobe-Japão 1995. b) Tombamento de estrutura devido à liquefação do solo no terremoto de Nigata-Japão 1964 (http://www.ngdc.noaa.gov/).

Figura 3.5 - Colapso de edificação devido a vibração excessiva no terremoto em Ica-Perú 2007 (http://www.elcomercio.pe).

Figura 3.6 - Registro de aceleração do sismo "El Centro (Califórnia 1940)" direção Norte-Sul (Clough e Penzien, 1995).

Figura 3.7 - FDEP gerada a partir de um acelerograma normalizado (Roehl, 2000).

Figura 3.8 - Fluxograma para a geração de um sismo artificial a partir de uma FDEP (Roehl, 2000).

Figura 4.1 - Modelo de torre esbelta T-1.

Figura 4.2 - Modelo de torre esbelta T-2.

Figura 4.3 - Modelo de pórtico de dois andares Po-2.

Figura 4.5 - Modelo de arco circular abatido.

Tabela 4-4. Três primeiras frequências e períodos de vibração da torre $\mathrm{T}-1$ 
Figura 4.6 - Variação do deslocamento horizontal no topo da torre

T-1 no

tempo com métodos Runge-Kutta explícitos.

Figura 4.7 - Variação do deslocamento horizontal no topo da torre

T-1 no tempo com os métodos de RKG4 implícito e Newmark.

$A=0.4 \mathrm{~g}, \mathrm{C}=0.25 \mathrm{M}, \omega=5.8 \mathrm{rad} / \mathrm{s}$.

Figura 4.8 - Deslocamento horizontal do ponto B em Po-2. A=0.4g, $\mathrm{C}=0.25 \mathrm{M}, \omega=7.86 \mathrm{rad} / \mathrm{s}$.

Figura 4.10 - Deslocamento horizontal do ponto B em T-2. A=0.4g, $\mathrm{C}=0.25 \mathrm{M}, \omega=5.1 \mathrm{rad} / \mathrm{seg}$.

Figura 4.11 - Deslocamento horizontal do ponto B em Po-6. A = 0.4g, $C=0.25 \mathrm{M}, \omega=2.3 \mathrm{rad} / \mathrm{seg}$.

Figura 4.12 - Deslocamento horizontal máximo de B em T-2 na fase permanente vs. frequência de excitação. $A=0.4 \mathrm{~g}, \mathrm{C}=0.25 \mathrm{M}$.

Figura 4.13 - Deslocamento horizontal máximo de B em Po-6 na fase permanente vs. frequência de excitação. $A=0.4 \mathrm{~g}, C=0.25 \mathrm{M}$.

Figura 4.14 - Deslocamento vertical de B vs. t em Ac-1. $A=0.8 g$, $C=0.50 \mathrm{M} . \mathrm{P}=0.0 \mathrm{kN}, \omega=1.95 \mathrm{rad} / \mathrm{s}$. Base rígida.

Figura 4.15 - Deslocamento vertical de B em Ac-1 vs. t. $C=0.5 \mathrm{M}, \omega=$ $0.80 \mathrm{rad} / \mathrm{s}, \mathrm{P}=0.2 \mathrm{kN}$.

Figura 4.16 - Deslocamento vertical de B em Ac-1 vs. t. C = 0.75M, $\omega=0.80 \mathrm{rad} / \mathrm{s}$. Duração da excitação de base $\mathrm{Tg}=15 \mathrm{~s}$.

Figura 4.17 - Relação P vs. deslocamento vertical de B em Ac-1 na fase permanente. $A=0.4 \mathrm{~g}, C=0.75 \mathrm{M}, \omega=0.80 \mathrm{rad} / \mathrm{s}$. Duração da excitação de base $\mathrm{Tg}=15 \mathrm{~s}$.

Figura 4.18 - Relação P vs. Deslocamento vertical de B em Ac-1 na fase permanente. $C=0.75 \mathrm{M}, \omega=0.80 \mathrm{rad} / \mathrm{s}$; duração da excitação de base $\mathrm{Tg}=15 \mathrm{~s}$.

Figura 4.19 - Deslocamento horizontal máximo de B em T-1 na fase permanente vs. frequência de excitação. $A=0.4 \mathrm{~g}, \mathrm{C}=0.25 \mathrm{M}$.

Figura 4.20 - Deslocamento horizontal máximo de B em Po-6 na fase permanente vs. frequência de excitação. $A=0.4 \mathrm{~g}, C=0.25 \mathrm{M}$.

Figura 4.21 - Deslocamento horizontal máximo de B em T-2 na fase permanente vs. A. $\omega=5.19 \mathrm{rad} / \mathrm{s}, \mathrm{C}=0.25 \mathrm{M}, \mathrm{k}_{\mathrm{r}}=$ infinito (rígido). 
Figura 4.22 - Deslocamento horizontal máximo de B em Po-6 na fase

permanente $\times A$. $\omega=1.95 \mathrm{rad} / \mathrm{s}, \mathrm{C}=0.25 \mathrm{M}, \mathrm{k}_{\mathrm{r}}=10^{6} \mathrm{kNm} / \mathrm{rad}$.

Figura 4.23 - Deslocamento vertical de B em Ac-1 x t. A=0.4g,

$\mathrm{C}=0.75 \mathrm{M}, \omega=5$. $0 \mathrm{rad} / \mathrm{s}, \mathrm{P}=0.2 \mathrm{kN}$. $\mathrm{k}_{\mathrm{h}}=$ rígido. Duração da

excitação de base $\mathrm{Tg}=15 \mathrm{~s}$.

Figura 4.24 - Deslocamento vertical de $B$ em $A c-1$ vs. t. $A=0.4 g$, $\mathrm{C}=0.75 \mathrm{M}, \omega=5$. Orad/s, $\mathrm{P}=0.2 . \mathrm{k}_{\mathrm{r}}=$ rígido. Duração da excitação de base $\mathrm{Tg}=15 \mathrm{~s}$.

Figura 4.25 - Deslocamento horizontal máximo de B em Ac-1 na fase permanente vs. frequência de excitação. $A=0.4 \mathrm{~g}, \mathrm{C}=0.25 \mathrm{M}, \mathrm{P}=0$, $k_{h}=$ infinito.

Figura 4.26 - Deslocamento horizontal de B em T-1 x t. A=0.4g, $\mathrm{C}=0.25 \mathrm{M}, \mathrm{k}_{\mathrm{r}}=10^{11} \mathrm{kNm} / \mathrm{rad}, \omega=5.40 \mathrm{rad} / \mathrm{s}, \mathrm{Mp}=160 \mathrm{MNm}$.

Figura 4.27 - Relação Momento vs. Rotação na base. $A=0.4 \mathrm{~g}$, $\mathrm{C}=0.25 \mathrm{M}, \mathrm{k}_{\mathrm{r}}=10^{11} \mathrm{kNm} / \mathrm{rad}, \omega=5.40 \mathrm{rad} / \mathrm{s}, \mathrm{Mp}=160 \mathrm{MNm}$.

Figura 4.28 - Sismo artificial gerado a partir do espectro de potência do sismo "El centro (1940)" direção Norte-Sul.

Figura 4.29 - Superposição de acelerogramas do sismo artificial e o sismo "El Centro".

Figura 4.30 - Comparação entre espectros de densidade de potência. 125

Figura 4.31 - Deslocamento horizontal no tempo do topo do sistema Po-6. $C=0.25 \mathrm{M}, \mathrm{k}_{\mathrm{r}}=10^{11} \mathrm{kNm} / \mathrm{rad}$. ts $=50 \mathrm{seg}$, tif $=5 \mathrm{seg}$, tff $=40 \mathrm{seg}$.

Figura 4.32 - Deslocamento horizontal de B em Po-6 x t, sismo Artificial. $\mathrm{C}=0.25 \mathrm{M}, \mathrm{k}_{\mathrm{r}}=10^{7} \mathrm{kNm} / \mathrm{rad}$, ts $=50 \mathrm{seg}$, tif $=5 \mathrm{seg}$.

Figura 4.33 - Deslocamento horizontal de B em T-1 x t, sismo Artificial. $\mathrm{C}=0.25 \mathrm{M}, \mathrm{k}_{\mathrm{r}}=10^{11} \mathrm{kNm} / \mathrm{rad}$. ts $=55 \mathrm{~s}$, tif $=5 \mathrm{~s}$, tff $=45 \mathrm{~s}$. $\mathrm{Mp}=80 \mathrm{MNm}$.

Figura 4.34 - Relação Momento x Rotação na base. Sismo artificial.

$\mathrm{C}=0.25 \mathrm{M}, \mathrm{k}_{\mathrm{r}}=10^{11} \mathrm{kNm} / \mathrm{rad}$, ts $=55 \mathrm{~s}$, tif $=5 \mathrm{~s}, \mathrm{tff}=45 \mathrm{~s}, \mathrm{Mp}=80 \mathrm{MNm} .128$

Figura 5.1 - Variação do deslocamento no tempo de um sistema de um grau de liberdade submetido a uma carga harmônica com frequência de excitação $\omega$; fase transiente e fase permanente.

Figura 6.1 - Pilar engastado e livre, submetido a deslocamento harmônico de base. 
Figura 6.2 - Deslocamento horizontal vs. tempo, $\mathrm{Ag}=0.4 \mathrm{~g}, \omega=$ $9.16 \mathrm{rad} / \mathrm{s}$.

Figura 6.3 - Norma da amplitude do deslocamento horizontal máximo no

topo do elemento vs. frequência, $\mathrm{Ag}=0.4 \mathrm{~g}$.

Figura 6.5 - Norma da amplitude do deslocamento horizontal máximo no topo vs. frequência de excitação.

Figura 6.6 - Sistema de 1 grau de liberdade com rigidez não linear não amortecido.

Figura 6.7 - Sistema de 1 grau de liberdade não amortecido com rigidez não linear.

Figura 6.8 - Norma do deslocamento horizontal máximo no topo vs. frequência.

Figura 6.9 - Arco abatido bi-engastado submetido a movimento harmônico vertical.

Figura 6.10 - Norma da amplitude vertical no meio do vão vs. frequência. $A=1.0 \mathrm{~g}, L=100 \mathrm{~m}$.

Figura 6.11 - Geometria de galpão com cobertura a duas águas.

Figura 6.12 - Primeiro e terceiro modos de vibração natural. Modos assimétricos.

Figura 6.13 - Segundo e quarto modo de vibração natural. Modos simétricos.

Figura 6.14 - Amplitude de vibração horizontal no meio do vão vs. frequência de excitação. Agx $=0.8 \mathrm{~g}$.

Figura 6.15 - Amplitude de vibração vertical no meio do vão vs.

frequência de excitação. Agy $=0.8 \mathrm{~g}$.

Figura 6.16 - Amplitude de vibração horizontal e vertical no meio do vão vs. frequência de excitação. Agx $=0.8 \mathrm{~g}$, Agy $=0.667 \mathrm{Agx}$.

Figura 6.17- Geometria de galpão com cobertura em arco circular. (dimensões em metros)

Figura 6.18 - Primeiro e terceiro modos de vibração natural. Modos não simétricos.

Figura 6.19 - Segundo e quarto modos de vibração natural. 
Figura 6.20 - Amplitude de vibração horizontal no meio do vão vs.

Frequência. $\operatorname{Agx}=0.8 \mathrm{~g}$.

Figura 6.21 - Amplitude de vibração vertical no meio do vão vs.

Frequência. Agy $=0.8 \mathrm{~g}$.

Figura 6.22 - Amplitude de vibração horizontal e vertical no meio do vão vs. Frequência. Agx $=0.8 \mathrm{~g}$, Agy $=0.66 \mathrm{Agx}$.

Figura 7.1 - Registo de aceleração e FDEP. a) Aceleração do sismo Kobe, componente Leste-Oeste. b) FDEP sismo Kobe. c) Aceleração do sismo El Centro, componente Norte-Sul. d) FDEP sismo El Centro. 168 Figura 7.2 - Registo de aceleração artificiais gerados a partir do sismo El Centro.

Figura 7.3 - Registo de aceleração artificiais gerados a partir do sismo Kobe.

Figura 7.4 - Geometria e parâmetros de pórtico plano PT-1.

Parâmetros da mola elasto-plástica:

$\mathrm{k} r=5 * 1010 \mathrm{Nm} / \mathrm{rad}, \mathrm{kep}=0.1 * \mathrm{k} r, \mathrm{Mp}=105 \mathrm{Nm}$.

Figura 7.5 - Resposta de deslocamento e aceleração no centro do vão central. Sismo Kobe (1995).

Figura 7.6 - Resposta de deslocamento e aceleração no centro do vão. Sismo El Centro (1940).

Figura 7.7 - Relação momento-rotação no apoio elasto-plástico.

Figura 7.8 - Resposta de deslocamento e aceleração horizontal no topo de PT-1. SAKB1.

Figura 7.9 - Resposta de deslocamento e aceleração no centro do vão.

Figura 7.10 - Relação momento rotação no apoio elasto-plástico.

Figura 7.11 - Superior: FDEP com concentração de potência nas regiões de ressonância. Inferior: Amplitude de vibração horizontal no topo de PT-1 sob aceleração harmônica com componente horizontal e vertical.

Figura7.12 - Aceleração do sismo SAPCP1.

Figura 7.13 - Deslocamento e aceleração horizontal do topo de PT-1 submetido ao sismo artificial SAPCP1. 
Figura 7.14 - Geometria e propriedades de galpão a duas águas

GPD-1. Parâmetros da mola elasto-plástica:

$\mathrm{k} r=5 * 1010 \mathrm{Nm} / \mathrm{rad}, \mathrm{kep}=0.1 * \mathrm{k} r, \mathrm{Mp}=105 \mathrm{Nm}$.

185

Figura 7.15 - Deslocamento horizontal do meio do vão

(topo da estrutura).

186

Figura 7.16 -. Relação momento-rotação no apoio elasto-plástico. 187

Figura 7.17 - Deslocamento horizontal no meio do vão central. 188

Figura 7.18 - Relação momento rotação no apoio elasto-plástico. 189

Figura 7.19 - Superior: FDEP com concentração de potência nas

regiões de ressonância. Inferior: Amplitude de vibração horizontal

no topo de PT-1 sob carga harmônica com aceleração horizontal e vertical.

Figura 7.20 - Aceleração do sismo SAPCG1.

Figura 7.21 - Deslocamento e aceleração horizontal do topo de GPD-1 submetido ao sismo artificial SAPCP1.

Figura 7.21 - Geometria e parâmetros de galpão a duas águas GPA-1.

Parâmetros da mola elasto-plástica:

$\mathrm{k} r=5 * 1010 \mathrm{Nm} / \mathrm{rad}$, $\mathrm{kep}=0.1 * \mathrm{k} r \mathrm{Mp}=105 \mathrm{Nm}$.

Figura 7.24 - Superior: FDEP com concentração de potência nas regiões de ressonância.

Figura 7.24 - Deslocamento horizontal no meio do no topo de GPA-1, com sismo replicado.

Figura 7.25 - Deslocamento horizontal no meio do no topo de GPA-1, sismos sucessivos alternados. 


\section{Listas de Tabelas}

Tabela 2-1. Estrutura do Arranjo de coeficientes de Butcher

Tabela 2-2. Arranjo de Butcher do método clássico de RK4

(Butcher,2003)

Tabela 2-3. Coeficientes de Niström de sexta ordem (Butcher,2003)

Tabela 2-4. Arranjo de coeficientes para o método de RKF45

(Butcher,2003)

Tabela 2-5. Arranjo de Butcher para o método de RKG4

Tabela 2-6. Esquema do algoritmo de Newmark para problema não linear

Tabela 2-7. Esquema do algoritmo de Runge-Kutta para problema não linear

Tabela 4-1. Parâmetros físicos e geométricos da estrutura T-1

Tabela 4-2. Parâmetros físicos e geométricos da estrutura T-2

Tabela 4-3. Parâmetros físicos e geométricos do arco Ac-1

105

Tabela 4-4. Três primeiras frequências e períodos de vibração da torre $\mathrm{T}-1$

Tabela 4-5. Tempo de processamento para análise de T-1

Tabela 4-6. $\Delta$ t máximo mantendo estabilidade na ressonância

Tabela 6-1. Frequências e períodos de vibração

Tabela 6-2. Primeiras quatro frequências vibração

Tabela 6-3. Primeiras quatro frequências

Tabela 7-1. Deslocamento e aceleração máxima no topo de PT-1

obtidos

com o sismo real e artificiais a partir de El Centro

Tabela 7-2. Deslocamento e aceleração máxima no topo de PT-1 obtidos com

o sismo real e artificiais a partir do registro de Kobe

Tabela 7-3. Deslocamento e aceleração máxima no topo de PT-1 
Tabela 7-4. Deslocamento e aceleração máxima no topo de GPD-1 obtidos

com o sismo real e sismos artificiais a partir do sismo El Centro

Tabela 7-5. Deslocamento e aceleração máxima no topo de GPD-1 obtidos

com o sismo real e sismos artificiais a partir do sismo Kobe

Tabela 7-6. Deslocamento e aceleração máxima no topo de GPD-1 obtidos com potências concentradas

Tabela 7-7. Deslocamento e aceleração máxima no topo de GPA-1 obtidos com sismos reais e artificiais a partir do sismo Kobe

Tabela 7-8. Deslocamento e aceleração máxima no topo de GPA-1 btidos com o sismo real e artificiais a partir do sismo El Centro

Tabela 7-9. Deslocamento e aceleração máxima no topo de GPD-1 obtidos com potências concentradas 


\section{Lista de Símbolos}

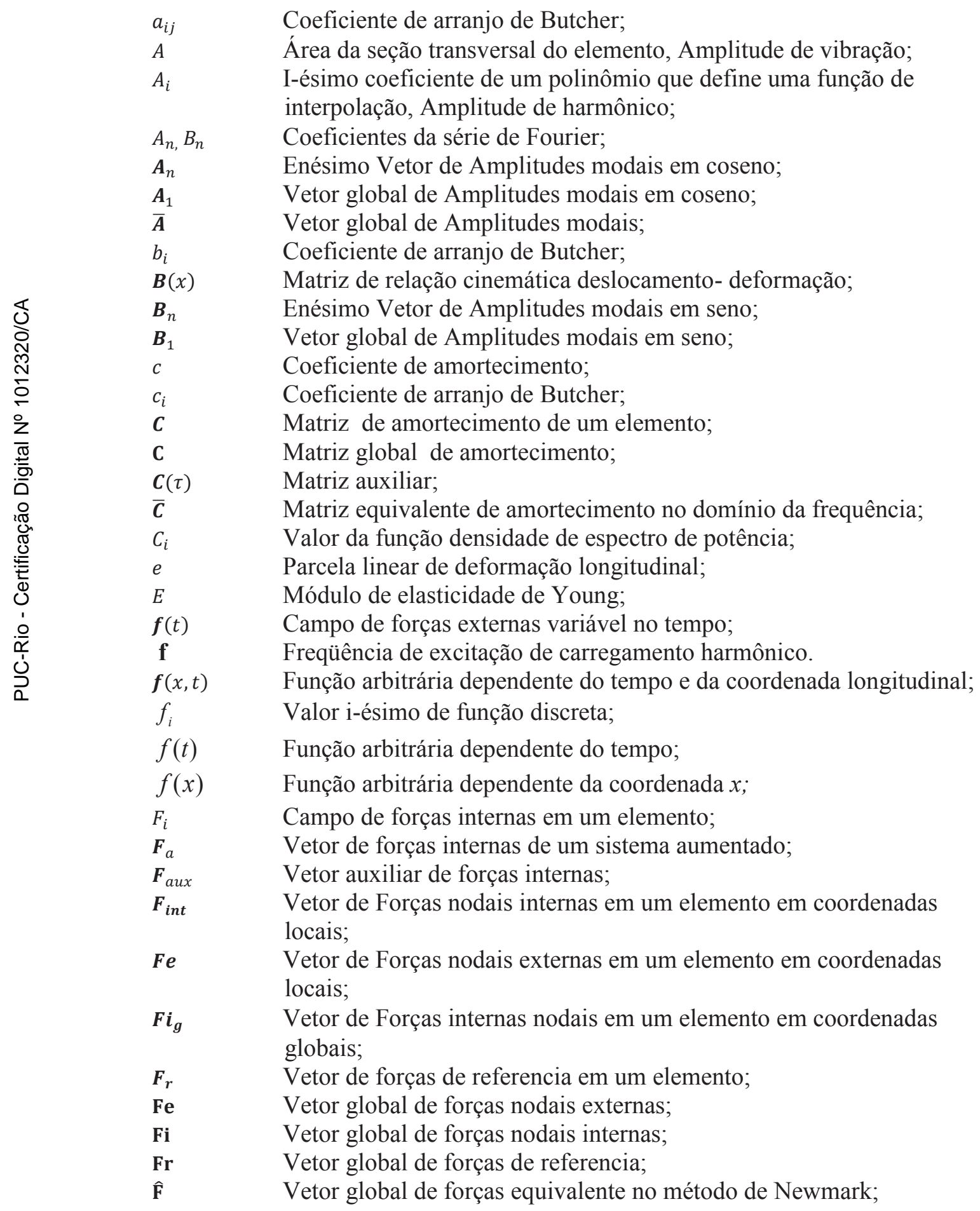




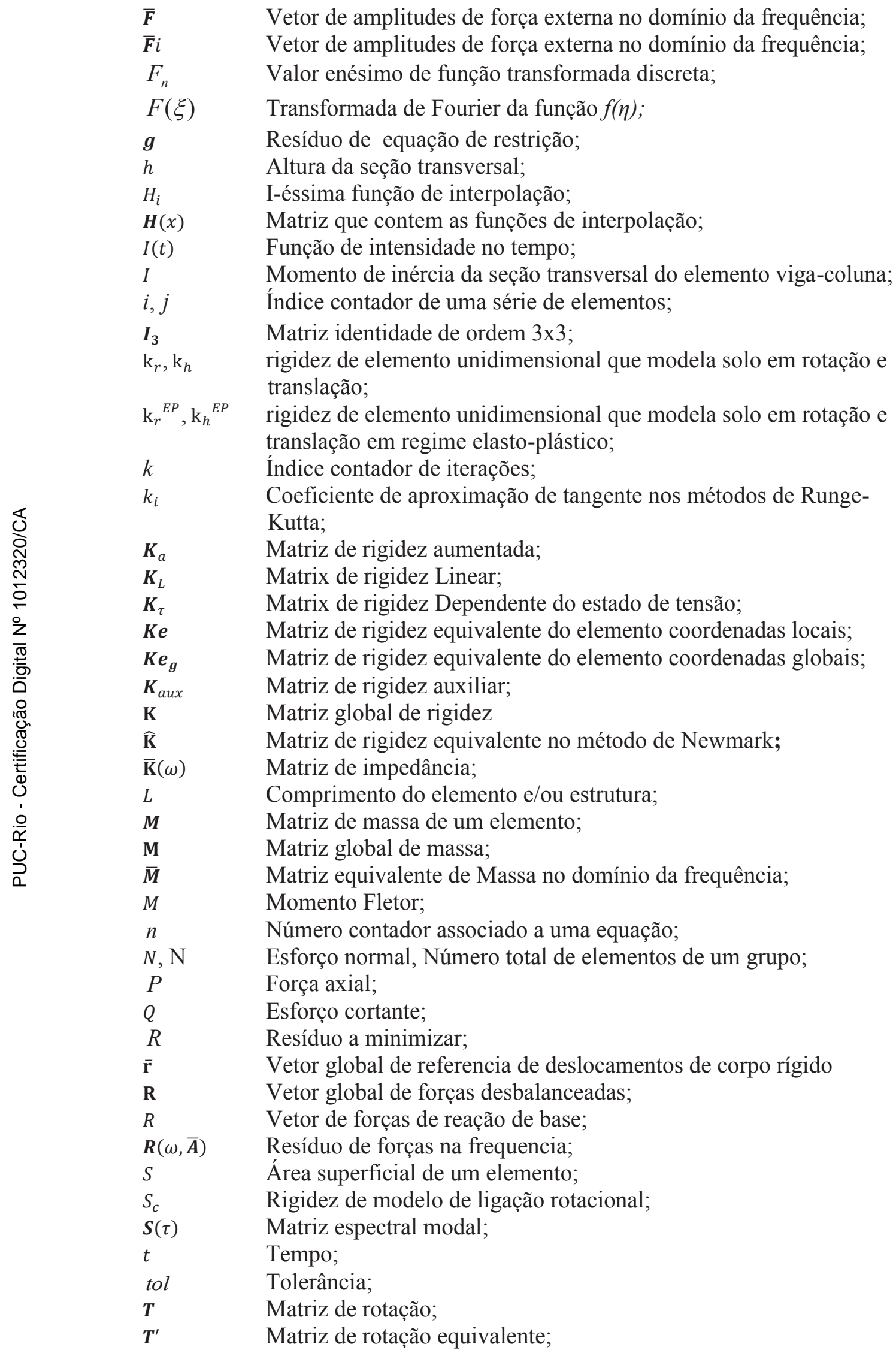




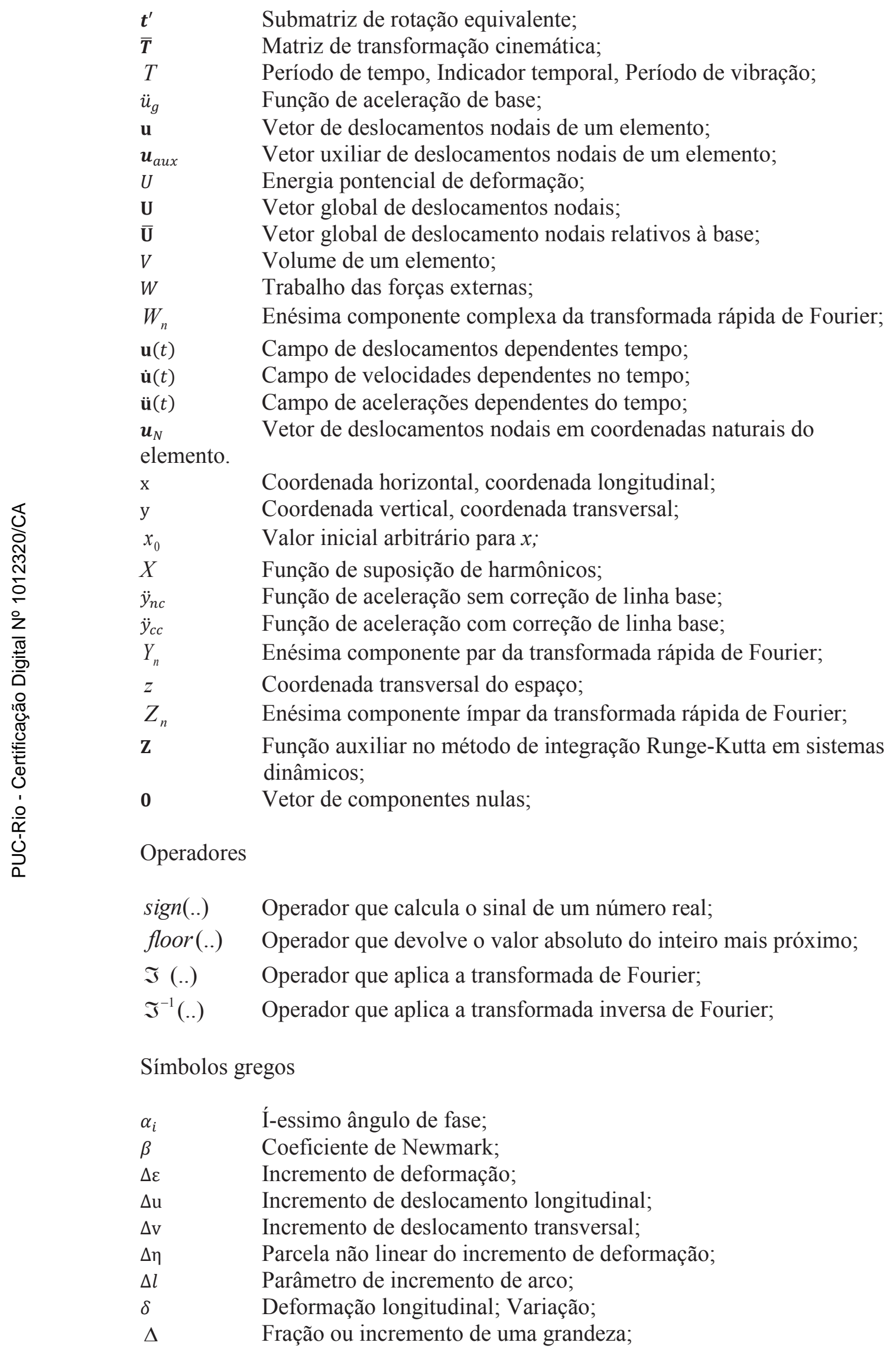




$\begin{array}{ll}\lambda & \text { Parâmetro de carga; } \\ \xi & \text { Taxa de amortecimento; } \\ \theta & \text { Deformação rotacional; } \\ \gamma & \text { Coeficiente de Newmark; } \\ \rho & \text { Densidade de material; } \\ \Delta \Pi & \text { Variação da energia potencial total; } \\ \tau & \text { Tensor de tensões, coordenada de tempo periódico } \\ \mu & \text { Coeficiente de proporcionalidade; } \\ \varphi & \text { Coeficiente de proporcionalidade no método do controle de arco; } \\ \phi_{f f}(\omega) & \text { Função densidade de espectro de potência; } \\ \phi & \text { Rotação relativa entre elemento e ligação; } \\ \phi & \text { Vetor que define modo de vibração; } \\ \Psi & \text { Rotação de corpo rígido; } \\ \Psi & \text { Função arbitrária de ponderação; } \\ \omega & \text { Freqüência circular de vibração/excitação externa; }\end{array}$

Siglas

\author{
Ac-1 Modelo de arco abatido; \\ FDEP Função densidade espectro de Potencia de uma função no tempo; \\ FFT Transformada rápida de Fourier; \\ GPD-I Modelo de galpão a duas águas; \\ GPA-I Modelo de galpão com cobertura em arco; \\ MBH Método do Balanço Harmônico; \\ Po-2 Modelo de pórtico de dois andares; \\ PT-I Modelo de pórtico plano de um andar; \\ Po-6 Modelo de pórtico de seis andares; \\ PK-II Segundo tensor de Piola-Kirchhof; \\ RLT Referencial Lagrangiano Total; \\ RLA Referencial Lagrangiano Atualizado; \\ T-1 Modelo de torre esbelta; \\ T-2 Modelo torre esbelta com massa concentrada no topo;
}


"Engineers ... are not superhuman. They make mistakes in their assumptions, in their calculations, in their conclusions. That they make mistakes is forgivable; that they catch them is imperative. Thus it is the essence of modern engineering not only to be able to check one's own work but also to have one's work checked and to be able to check the work of others."

Henry Petrosky 